Revista Bioética

\title{
PESQUISA
}

\section{Satisfação de usuários das unidades de saúde em Coari, Amazonas}

Ana Felisa Hurtado Guerrero ${ }^{1}$, Ana Possidonio Alves ${ }^{1}$, Gilmara Lima Libório ${ }^{1}$, Joziane Vieira de Freitas ${ }^{1}$, José Camilo Hurtado Guerrero ${ }^{2}$

1. Escola de Ciências da Saúde, Universidade do Estado do Amazonas, Manaus/AM, Brasil. 2. Instituto de Biodiversidade e Floresta, Universidade Federal do Oeste do Pará, Santarém/PA, Brasil.

\section{Resumo}

Este trabalho analisou a qualidade da atenção em unidades básicas de saúde de Coari, Amazonas, Brasil. Trata-se de estudo descritivo, quantitativo, de caráter avaliativo, que utilizou questionário validado do Programa Nacional de Avaliação de Serviços de Saúde de 2015. Os dados foram coletados em três unidades, no segundo semestre de 2016. Os resultados apontam que a maioria dos entrevistados era do sexo feminino, casada, com 40 anos de idade ou mais, autodeclarada parda, com ensino médio completo, moradora da área urbana e de baixa renda. $\mathrm{O}$ atendimento na recepção foi considerado bom, mas os usuários se mostraram insatisfeitos com a disponibilidade de medicamentos. Na avaliação da limpeza do ambiente, as porcentagens ficaram entre bom e regular, e a estrutura física dos estabelecimentos de saúde foi considerada boa.

Palavras-chave: Humanização da assistência. Satisfação do paciente. Avaliação em saúde.

\section{Resumen}

\section{Satisfacción de usuarios de las unidades de salud de Coari, Amazonas, Brasil}

Este estudio analizó la calidad de la atención ofrecida por las unidades básicas de salud de Coari, Amazonas, Brasil. Se trata de una investigación cuantitativa, descriptiva y evaluativa, que utilizó el cuestionario validado del Programa Nacional de Evaluación de Servicios de Salud 2015. Se recolectaron los datos en tres unidades, en el segundo semestre del 2016. Los resultados indicaron que la mayoría de los encuestados eran mujeres, casadas, con 40 años o más de edad, autodeclaradas pardas, con la secundaria completa, residentes en la zona urbana y de bajos ingresos. Los usuarios consideraron el servicio de recepción bueno, pero no estaban satisfechos con la falta de disponibilidad de medicamentos. En cuanto a la limpieza del ambiente, los porcentajes quedaron entre bueno y regular, y la estructura de los establecimientos de salud se evaluó como buena.

Palabras clave: Humanización de la atención. Satisfacción del paciente. Evaluación en salud.

\begin{abstract}
User satisfaction with health centers in Coari, Amazonas, Brazil

This study analyzed the quality of care in health centers in Coari, Amazonas, Brazil. This is a descriptive, quantitative, and evaluative research that used a validated questionnaire from the 2015 National Health Services Evaluation Survey. Data were collected in three centers in the second half of 2016. The results show that most interviewees were female, married, 40 years of age or older, self-declared as brown, with high school degree and low income, living in the urban area. Users considered the reception service in the units good, but were dissatisfied with the availability of medicines. Regarding cleanliness of the environment, the percentages ranged from good to fair, and the physical structure of health facilities was considered good.

Keywords: Humanization of assistance. Patient satisfaction. Health evaluation.
\end{abstract}


A avaliação é etapa importante para revisar e reorientar ações de saúde. Ela é ferramenta fundamental que deve ser incorporada a fim de aperfeiçoar o sistema. Seu objetivo é verificar a eficiência, eficácia e efetividade de estruturas, processos e resultados, considerando vulnerabilidades no acesso e a satisfação dos cidadãos ${ }^{1}$. A qualidade na assistência é indispensável, e pode ser julgada averiguando-se, entre outros aspectos, o contentamento dos usuários - item indireto de avaliação, mas importante para planejar os serviços ${ }^{2}$.

A satisfação pode ser entendida como a comparação entre a experiência e as expectativas dos usuários quanto ao desempenho e a qualidade do sistema, e avaliá-la implica apontar dificuldades tanto na comunicação com a rede de serviços de saúde quanto na continuidade do atendimento e integralidade do cuidado $^{3}$. Quando as expectativas não são atendidas, a consequência é a insatisfação, que pode levar o usuário a abandonar o tratamento ou, quando dispõe de condições financeiras, procurar o sistema privado ${ }^{3}$.

O grau de satisfação ou insatisfação do usuário se relaciona a fatores como tipo de atendimento, equipamentos, medicamentos, infraestrutura e materiais, ventilação de ambientes fechados e conforto. O contentamento é o verdadeiro atributo da ideia de qualidade e deve ser visto como objetivo em si, não como meio de fazer o indivíduo concordar com o tratamento ${ }^{4}$.

A melhoria da assistência do ponto de vista da satisfação indica respeito à dignidade dos sujeitos e gera informações eficazes para a gestão ${ }^{5}$. Assim, gestores e trabalhadores podem medir com mais precisão as reais necessidades dos pacientes, procurando desenvolver continuamente serviços e processos de trabalho que os possam satisfazer. Nesse sentido, este estudo teve por objetivo avaliar a satisfação de usuários com a atenção ofertada nas unidades básicas da Estratégia Saúde da Família no município de Coari/AM.

\section{Método}

Trata-se de estudo descritivo, quantitativo, avaliativo, baseado no Programa Nacional de Avaliação de Serviços de Saúde (Pnass) ${ }^{6}$, proposto pelo Ministério da Saúde em 2004. O foco da pesquisa é o Questionário da Avaliação da Satisfação dos Usuários, instrumento com questões fechadas que apura o contentamento com a assistência recebida. O modelo avalia as seguintes dimensões: estrutura, processo, resultado, produção do cuidado, gerenciamento de risco e satisfação dos pacientes.

A ferramenta abrange o perfil sociodemográfico dos usuários (idade, sexo, escolaridade, cor, ocupação, estado civil e renda familiar) e variáveis de acesso ao serviço de saúde (atendimento na recepção, educação, respeito e interesse dos profissionais, atendimento da equipe, disponibilidade de medicamentos e limpeza do ambiente, ética no atendimento e avaliação dos estabelecimentos).

Os entrevistadores receberam treinamento prévio e tiveram seu desempenho avaliado em estudo-piloto, fundamental para o trabalho de campo. A finalidade desse estudo-piloto foi testar o questionário e treinar os entrevistadores para a coleta de dados, buscando definir a abordagem mais adequada para a população estudada. Foram verificadas dificuldades dos participantes para compreender alguns termos do instrumento, o que levou os pesquisadores a pensar alternativas para explicar os conceitos.

Os dados foram coletados no município de Coari/AM entre outubro e dezembro de 2016, em três unidades básicas de saúde (UBS): Dona Luzia Tenório, localizada no bairro Santa Helena (área menos privilegiada); Ribeirinha, no bairro Tauá-Mirim (área rural); e Chico Enfermeiro, no bairro Chagas Aguiar (área mais favorecida). Os participantes foram esclarecidos quanto ao objetivo da pesquisa e assinaram termo de consentimento livre e esclarecido. Foram entrevistados 329 usuários com mais de 18 anos de idade em entrevistas com duração média de 30 a 35 minutos.

Utilizaram-se na análise medidas de frequência absoluta e relativa (\%), e foram eliminadas entrevistas de participantes que não souberam informar ou não responderam algum dado. O banco de dados foi organizado no programa Microsoft Office Excel versão 2010, e as variáveis foram analisadas nos softwares Minitab 14, SPSS 20 e Epi Info 7.

O estudo foi aprovado pelo comitê de ética em pesquisa da Universidade do Estado do Amazonas e pela Secretaria Municipal de Saúde de Coari. O anonimato e a confidencialidade das informações foram garantidos conforme preconizado pela Resolução do Conselho Nacional de Saúde 466/2012 ${ }^{7}$.

\section{Resultados}

Responderam ao questionário 329 usuários: 122 $(37,1 \%)$ da UBS Dona Luzia Tenório, 129 (39,2\%) da UBS Chico Enfermeiro e 78 (23,7\%) da UBS Ribeirinha. A maioria dos entrevistados (78,4\%) era do sexo feminino, com 40 anos de idade ou mais $(28,9 \%)$, casada $(33,7 \%)$ e de cor parda $(67,9 \%)$. Quanto ao nível de escolaridade, $28,3 \%$ haviam completado o ensino médio. A maior parte dos participantes (76,3\%) vinha da área urbana, e 46,3\% declararam ter renda menor que um salário mínimo (Tabela 1). 
Tabela 1. Características sociodemográficas e econômicas de usuários das unidades básicas de saúde (Coari/AM, 2016)

\begin{tabular}{|c|c|c|}
\hline Característica & $n$ & $\%$ \\
\hline \multicolumn{3}{|l|}{$\overline{\text { Sexo }}$} \\
\hline Masculino & 71 & 21,6 \\
\hline Feminino & 258 & 78,4 \\
\hline \multicolumn{3}{|l|}{ Faixa etária } \\
\hline Menos de 19 anos & 41 & 12,5 \\
\hline 20 a 24 anos & 56 & 17,0 \\
\hline 25 a 29 anos & 66 & 20,1 \\
\hline 30 a 34 anos & 35 & 10,6 \\
\hline 35 a 39 anos & 36 & 10,9 \\
\hline 40 anos ou mais & 95 & 28,9 \\
\hline \multicolumn{3}{|l|}{ Estado civil } \\
\hline Casado(a) & 111 & 33,7 \\
\hline Solteiro(a) & 93 & 28,3 \\
\hline Viúvo(a) & 18 & 5,5 \\
\hline Divorciado(a) & 4 & 1,2 \\
\hline Separado(a) & 6 & 1,8 \\
\hline União estável & 97 & 29,5 \\
\hline \multicolumn{3}{|l|}{ Cor } \\
\hline Branco(a) & 49 & 15,1 \\
\hline Preto(a) & 36 & 11,1 \\
\hline Amarelo(a) & 11 & 3,4 \\
\hline Pardo(a) & 220 & 67,9 \\
\hline Indígena & 8 & 2,5 \\
\hline Não sabe/não respondeu* & 5 & - \\
\hline \multicolumn{3}{|l|}{ Escolaridade } \\
\hline Não sabe ler/escrever & 21 & 6,4 \\
\hline Alfabetizado & 31 & 9,4 \\
\hline Nível fundamental incompleto & 87 & 26,4 \\
\hline Nível fundamental completo & 21 & 6,4 \\
\hline Nível médio incompleto & 47 & 14,3 \\
\hline Nível médio completo & 93 & 28,3 \\
\hline Superior incompleto & 17 & 5,2 \\
\hline Superior completo & 8 & 2,4 \\
\hline Especialização/residência & 4 & 1,2 \\
\hline \multicolumn{3}{|l|}{ Renda familiar } \\
\hline Não tem renda & 74 & 22,6 \\
\hline Menos de 1 salário mínimo & 152 & 46,3 \\
\hline Entre 1 e 2 salários mínimos & 89 & 27,1 \\
\hline Mais de 2 e até 5 salários mínimos & 12 & 3,7 \\
\hline Mais de 5 e até 10 salários mínimos & 1 & 0,3 \\
\hline Não sabe/não respondeu* & 1 & - \\
\hline \multicolumn{3}{|l|}{ Zona } \\
\hline Urbana & 251 & 76,3 \\
\hline Rural & 78 & 23,7 \\
\hline
\end{tabular}

*Frequência mostrada, mas não incluída na análise
Quanto à satisfação dos pacientes, predominaram boas avaliações na maioria dos indicadores. Quando questionados sobre o atendimento na recepção, $6,7 \%$ o avaliaram como muito bom, $54 \%$ como bom, $23 \%$ como regular, $14,1 \%$ como ruim, e $2,1 \%$ como muito ruim (Gráfico 1 ). Três participantes não responderam.

Gráfico 1. Avaliação do atendimento da recepção em unidades básicas de saúde (Coari/AM, 2016)

100

80

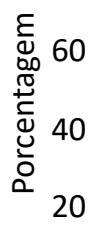

$22(6,7)$

0

Muito bom Bom Regular Ruim Muito ruim

Nas respostas referentes a educação, respeito e interesse demonstrados pela equipe de saúde, $79 \%$ dos usuários consideraram que os profissionais se mostraram educados, $80,2 \%$ responderam que foram respeitosos, e $62,6 \%$ interessados. Quanto ao atendimento, $61,7 \%$ o consideraram bom. Já quanto à disponibilidade de medicamentos, $47,7 \%$ se mostraram insatisfeitos, avaliando esse aspecto do serviço como muito ruim. Em relação à limpeza do ambiente, duas porcentagens quase se igualaram: bom $(42,6 \%)$ e regular $(41,6 \%)$. Os dados completos podem ser vistos na Tabela 2.

Quando questionados sobre o comportamento ético dos profissionais, $52,4 \%$ dos participantes consideraram esse aspecto do atendimento bom. Porém, vale ressaltar que percentual considerável apontou esse indicador como regular $(29,3 \%)$ e ruim (8,5\%) (Gráfico 2). Vinte e dois entrevistados não responderam a essa pergunta.

$\mathrm{Na}$ avaliação das unidades, 3,4\% dos participantes qualificaram a estrutura e o funcionamento como muito bons, e $42,5 \%$ como bom. Porém, 37,8\% consideraram esses aspectos regulares, $12,3 \%$ ruins, e $4 \%$ muito ruins (Gráfico 3). Essas últimas opiniões, que somam $54,1 \%$ do total das respostas, indicam grande insatisfação. Quatro entrevistados não responderam a essa pergunta. 
Tabela 2. Satisfação de usuários de unidades básicas de saúde (Coari/AM, 2016)

\begin{tabular}{|c|c|c|}
\hline Característica & n & $\%$ \\
\hline \multicolumn{3}{|c|}{ Os profissionais se mostraram educados? } \\
\hline Sim & 260 & 79,0 \\
\hline Não & 69 & 21,0 \\
\hline \multicolumn{3}{|c|}{ Os profissionais se mostraram respeitosos? } \\
\hline Sim & 264 & 80,2 \\
\hline Não & 65 & 19,8 \\
\hline \multicolumn{3}{|c|}{ Os profissionais se mostraram interessados? } \\
\hline Sim & 206 & 62,6 \\
\hline Não & 123 & 37,4 \\
\hline \multicolumn{3}{|l|}{ Atendimento da equipe } \\
\hline Muito bom & 30 & 9,1 \\
\hline Bom & 203 & 61,7 \\
\hline Regular & 79 & 24,0 \\
\hline Ruim & 12 & 3,7 \\
\hline Muito ruim & 5 & 1,5 \\
\hline \multicolumn{3}{|c|}{ Disponibilidade de medicamentos } \\
\hline Muito bom & 5 & 1,5 \\
\hline Bom & 31 & 9,5 \\
\hline Regular & 51 & 15,6 \\
\hline Ruim & 84 & 25,7 \\
\hline Muito ruim & 156 & 47,7 \\
\hline Não sabe/não respondeu* & 2 & - \\
\hline \multicolumn{3}{|l|}{ Limpeza do ambiente } \\
\hline Muito bom & 4 & 1,2 \\
\hline Bom & 140 & 42,6 \\
\hline Regular & 137 & 41,6 \\
\hline Ruim & 36 & 10,9 \\
\hline Muito ruim & 12 & 3,7 \\
\hline
\end{tabular}

*Frequência mostrada, mas não incluída na análise

Gráfico 2. Ética dos profissionais de unidades básicas de saúde (Coari/AM, 2016)

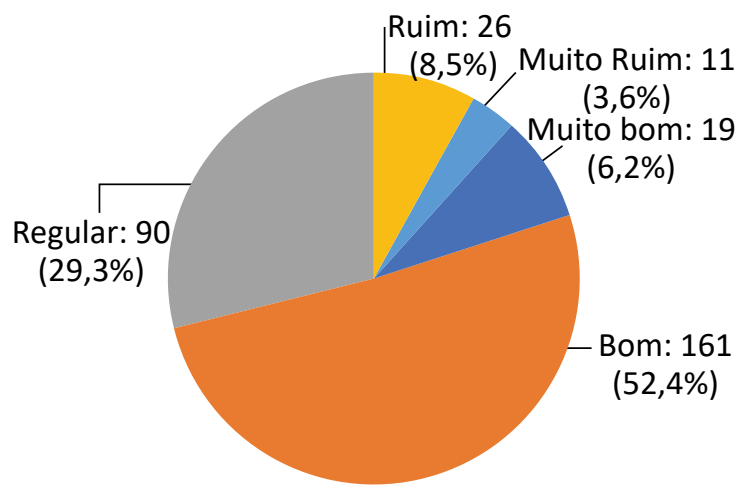

Gráfico 3. Avaliação da estrutura e funcionamento de unidades básicas de saúde (Coari/AM, 2016)

100

80

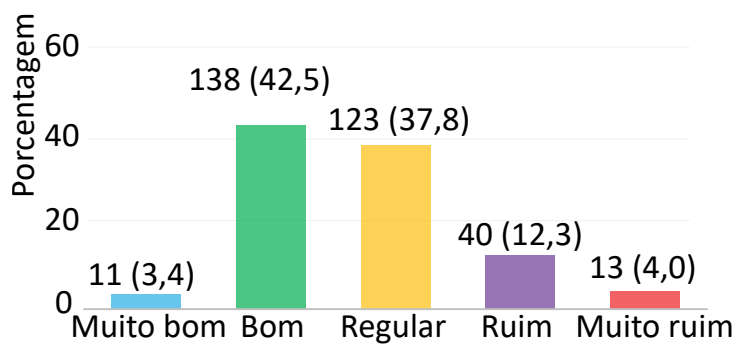

\section{Discussão}

$\mathrm{Na}$ amostra de outros estudos sobre satisfação de usuários de unidades de saúde ${ }^{4,6}$ também predominaram o sexo feminino, a escolaridade de nível médio e o estado civil "casado". Dado sociodemográfico interessante é que, segundo Gouveia ${ }^{8}$, usuários do Sistema Único de Saúde (SUS) com baixa escolaridade tendem a avaliar o sistema positivamente, enquanto pessoas com alta escolaridade o avaliam de forma negativa.

Quanto à compreensão dos usuários sobre a função das UBS, Garnelo e colaboradores ${ }^{9}$, em levantamento feito em 2012 para o Programa Nacional de Melhoria do Acesso e da Qualidade da Atenção Básica no Amazonas, apontaram que alguns usuários interpretam o acesso ao sistema como um direito, enquanto outros o veem como uma concessão. Estes últimos não se sentiam legitimados a exigir algo melhor e demonstravam gratidão pelo mero fato de haver assistência. Os mesmos pesquisadores constataram que, embora muitos usuários dessem notas baixas ao avaliar o cuidado, outros tendiam a atribuir notas altas ao serviço por temer que as UBS fossem fechadas ${ }^{9}$.

Outras questões podem ser levantadas: será que a delicada conjuntura política de Coari à época da pesquisa, com rotatividade de prefeitos e secretários de saúde, gerou certo desconforto nas pessoas, que, temendo retaliações, não se sentiram confortáveis para falar de forma aberta? É possível que usuários com maior escolaridade tenham mais conhecimento sobre a atenção à saúde em termos de direitos, enquanto os de baixa escolaridade têm noção menos clara da gratuidade do acesso? Ou será que, precisamente pelo fato de a população de baixa renda saber que o serviço é gratuito, aplica-se o dito popular na cidade "o que é de graça, ninguém pode 
reclamar, porque é dado"? Estudos futuros deverão analisar de maneira mais profunda essas questões.

Constatou-se também que as unidades foram procuradas majoritariamente pela população menos favorecida, o que confirma outros estudos ${ }^{10,11}$. A maioria dos usuários do SUS pertence a estratos de baixa renda, e fatores socioeconômicos são determinantes no uso desses serviços. Disso se depreende que a assistência pública, pautada no princípio de justiça social, continua devendo promover qualidade de vida para os mais pobres.

Neste estudo verificou-se percentual considerável de entrevistados que julgaram a ética dos profissionais como regular. Consideram-se problemas éticos no atendimento situações que geram desconforto ou prejuízos para a assistência, o paciente ou sua família ${ }^{11}$, por exemplo quando um profissional expõe o problema de saúde do doente a terceiros, podendo ocasionar abandono do tratamento. Montenegro e colaboradores ${ }^{12}$ identificaram como principais problemas éticos os relacionados a exposição do usuário, preconceito e discriminação. Além do sigilo, Nunes ${ }^{13}$, em estudo com enfermeiros de Portugal, apontou conflitos relacionados ao acompanhamento em fim de vida e ao respeito pelos direitos humanos.

O sigilo das informações é essencial. A escuta deve ser qualificada, correspondendo às demandas do cuidado, desde o momento da recepção até a alta. O acolhimento denota a postura, o compromisso e a responsabilidade dos trabalhadores com as necessidades de saúde do paciente ${ }^{14}$. Tal postura deve facilitar o cuidado, não o negligenciar, incluindo a diversidade de modo a estimular novos modos de cuidar e de organizar a assistência ${ }^{15}$. Com relação a esse aspecto, os usuários das UBS de Coari parecem estar satisfeitos.

Quanto à educação, ao respeito e ao interesse demonstrados pela equipe de saúde, a maioria dos entrevistados mostrou-se satisfeita, o que coincide com resultados do Pnass ${ }^{1}$. A forma como o profissional da saúde atende o usuário é importante, pois o mau atendimento desumaniza a relação, gerando desinteresse e insatisfação ${ }^{14}$. Por isso, o grau de contentamento do paciente tende a estar mais relacionado ao acolhimento ofertado do que a tecnologias de saúde e habilidades técnicas da equipe. É imprescindível para o doente contar com postura amigável e carinhosa de profissionais que demonstrem compreender as necessidades do momento ${ }^{16}$.

Porto, Schierholt e Costa ${ }^{17}$ observam que os profissionais de saúde identificam de maneira persistente o desejo do paciente em ser ouvido, percebido e reconhecido. Os usuários almejam relação que priorize aspectos mais triviais da comunicação humana, e não somente a formação técnica. Por isso, elementos subjetivos devem ser considerados ao se avaliar a qualidade da atenção. O bom relacionamento com o paciente, fundamental para sua satisfação, exige atendimento integral, centrado no respeito à subjetividade e às expectativas dos indivíduos. Assim, para os profissionais, são essenciais o comprometimento, a formação adequada, o comportamento ético e a capacidade de lidar com o tempo de consulta, bem como a boa infraestrutura dos serviços ${ }^{18}$.

Neste estudo, os usuários avaliaram positivamente a limpeza dos ambientes, corroborando os resultados de Santiago e colaboradores ${ }^{19}$. Esse indicador está de acordo com o preconizado pela Carta dos Direitos dos Usuários da Saúde ${ }^{20}$, que assegura ao cidadão o direito básico de ingressar dignamente no sistema de saúde público. De acordo com esse documento, o paciente deve esperar atendimento em lugar protegido, limpo e ventilado, com água potável e sanitários disponíveis, sem fumaça de cigarro e bebidas alcoólicas, de modo a contribuir para o bem-estar de todos ${ }^{20}$.

A disponibilidade de medicamentos nas unidades foi a dimensão mais mal avaliada. A escassez de remédios, um dos principais problemas da assistência em Coari na época, comprometia ações de prevenção, controle das doenças e continuidade do tratamento. O problema é agravado pelo fato de a maioria dos usuários do sistema provir de estratos de baixa renda, sem condições de arcar com os gastos da compra de medicamentos.

Por meio do princípio da integralidade, o SUS garante ações de promoção da saúde, prevenção de doenças, tratamento digno e reabilitação, assegurando a todo cidadão o direito de obter gratuitamente os medicamentos de que precisa. O Brasil passa por acelerada transição demográfica, caracterizada pelo alastramento de condições crônicas. Neste contexto, a assistência farmacêutica desempenha papel importante, considerando que uma das nove metas mundiais para controlar doenças crônicas não transmissíveis é garantir $80 \%$ de disponibilidade de tecnologias básicas e medicamentos essenciais, uma vez que o tratamento farmacológico apropriado reduz significativamente a morbimortalidade ${ }^{21}$.

Estudos têm constatado baixa disponibilidade de medicamentos-chave ${ }^{22} \mathrm{e}$, na falta do remédio necessário, a conduta mais adotada pelos médicos é analisar a possibilidade de substituí-lo ou de encaminhar o enfermo para a Farmácia Popular. Porém, 
Nascimento e colaboradores ${ }^{21}$ referem que apenas nove dos 50 medicamentos de uso contínuo verificados são fornecidos gratuitamente por esse programa. Os autores ainda apontam que as regiões Norte e Nordeste têm menor disponibilidade de medicamentos e menos farmacêuticos responsáveis técnicos, refrigeradores exclusivos e armários com chaves para armazenar as drogas ${ }^{21}$.

Segundo Helfer e colaboradores ${ }^{22}$, o comprometimento do acesso gratuito aos medicamentos, especialmente aos de uso contínuo, pode levar ao abandono do tratamento e a complicações do estado de saúde, prejudicar o orçamento familiar e, consequentemente, aumentar gastos com atendimentos ambulatoriais e internações. O problema é grave, visto que, como dito, o sistema público é a única forma de obter os fármacos necessários para a maior parte dos usuários.

Quanto à estrutura das unidades, percentual relevante dos entrevistados a considerou regular, mas a maioria das avaliações foi positiva. É possível, no entanto, que esse resultado se deva a baixa expectativa em relação ao serviço, como mostrado em outro estudo que sugere essa relação ${ }^{23}$. De qualquer modo, considerando os dados brutos, pôde-se observar que os usuários estavam satisfeitos com os serviços ofertados pelo SUS, mesmo em meio à crise que o município de Coari enfrentava na área saúde em 2016.

\section{Considerações finais}

A partir dos resultados obtidos, foi possível identificar que os usuários estavam satisfeitos com 0 atendimento das equipes das três unidades pesquisadas. Constatou-se, porém, que a maioria dos entrevistados havia completado apenas o ensino médio e ganhava em média menos de um salário mínimo, o que pode ter influenciado os resultados em razão de menor expectativa em relação aos serviços e desconhecimento de direitos básicos.

O fator de maior insatisfação foi a falta de medicamentos, que prejudica ações de prevenção e controle de doenças crônicas não transmissíveis, levando os usuários a peregrinar em busca de medicação em outras unidades. Esse problema também pode se relacionar aos contratempos que levam a população da zona rural a procurar atendimento na sede do município. Por outro lado, quanto às condições materiais das UBS (limpeza dos banheiros, corredores, recepção, salas de espera e consultórios), os participantes da pesquisa se mostraram satisfeitos.

Espera-se que este estudo contribua para transformar e melhorar a qualidade do atendimento aos usuários das unidades básicas de saúde de Coari.

\section{Referências}

1. Brasil. Ministério da Saúde. Pnass: Programa Nacional de Avaliação de Serviços de Saúde: resultado do processo avaliativo 2004-2006 [Internet]. Brasília: Ministério da Saúde; 2007 [acesso 8 ago 2019]. Disponível: https://bit.ly/30K7MDG

2. Véras MMS. A satisfação dos usuários do SUS com o serviço de ouvidoria em saúde de FortalezaCE [dissertação] [Internet]. Fortaleza: Universidade Federal do Ceará; 2005 [acesso 8 ago 2019]. Disponivel: https://bit.ly/32X3StX

3. Tahan-Santos E. Estratégia Saúde da Família: satisfação de usuários [dissertação] [Internet]. Ribeirão Preto: Universidade de São Paulo; 2011 [acesso 8 ago 2019]. Disponível: https://bit.ly/2WQqmsu

4. Hollanda E, Siqueira SAV, Andrade GRB, Molinaro A, Vaitsman J. Satisfação e responsividade em serviços de atenção à saúde da Fundação Oswaldo Cruz. Ciênc Saúde Coletiva [Internet]. 2012 [acesso 8 ago 2019];17(12):3343-52. DOI: 10.1590/S1413-81232012001200019

5. Santos MLMF, Cruz SS, Gomes-Filho IS, Soares JSP, Figueiredo ACMG, Coelho CM. Satisfação dos usuários adultos com a atenção em saúde bucal na estratégia de saúde da família. Cad Saúde Colet [Internet]. 2015 [acesso 8 fev 2019];23(2):163-71. DOI: 10.1590/1414-462X201500020057

6. Brasil. Ministério da Saúde. Pnass: Programa Nacional de Avaliação dos Serviços de Saúde [Internet]. Brasília: Ministério da Saúde; 2015 [acesso 8 fev 2019]. Disponível: https://bit.ly/3jvo6k6

7. Conselho Nacional de Saúde. Resolução CNS no 466, de 12 de dezembro de 2012. Aprova diretrizes e normas regulamentadoras de pesquisas envolvendo seres humanos. Diário Oficial da União [Internet]. Brasília, no 12, p. 59, 13 jun 2013 [acesso 11 fev 2019]. Seção 1. Disponível: https://bit.ly/20Vtf7a

8. Gouveia GC. Avaliação da satisfação dos usuários com o sistema de saúde brasileiro [tese] [Internet]. Recife: Fundação Oswaldo Cruz; 2009 [acesso 8 ago 2019]. Disponível: https://bit.ly/2ZTHTC6

9. Garnelo L, Vieira JMR, Souza M, Rocha ESC, Gonçalves MJF. Avaliação externa do PMAQ no Amazonas: experiência e narrativas sobre a implementação da Política Nacional de Atenção Básica. In: Fausto MCR, Fonseca HMS, organizadoras. Rotas da atenção básica no Brasil: experiências do trabalho de campo PMAQ AB [Internet]. Rio de Janeiro: Saberes; 2014 [acesso 11 fev 2019]. p. 61-89. Disponível: https://bit.ly/30lvro3 
10. Pavão ALB, Coeli CM, Lopes CS, Faerstein E, Werneck GL, Chor D. Uso de serviços de saúde segundo posição socioeconômica em trabalhadores de uma universidade pública. Rev Saúde Pública [Internet]. 2012 [acesso 8 mar 2019];46(1):98-103. DOI: 10.1590/S0034-89102012005000002

11. Levorato $C D$, Mello LM, Silva AS, Nunes AA. Fatores associados à procura por serviços de saúde numa perspectiva relacional de gênero. Ciênc Saúde Coletiva [Internet]. 2014 [acesso $18 \mathrm{mar}$ 2019];19(4):1263-74. DOI: 10.1590/1413-81232014194.01242013

12. Montenegro LC, Rénno HMS, Caram CS, Brito MJM. Problemas éticos na prática de profissionais de saúde em um hospital escola. Av Enferm [Internet]. 2016 [acesso 25 jan 2019];34(3):226-35. DOI: 10.15446/av.enferm.v34n3.45590

13. Nunes L. Problemas éticos identificados por enfermeiros na relação com usuários em situação crítica. Rev. bioét. (Impr.) [Internet]. 2015 [acesso 19 abr 2019];23(1):187-99. DOI: 10.1590/1983-80422015231059

14. Ramos DD, Lima MADS. Acesso e acolhimento aos usuários em uma unidade de saúde de Porto Alegre, Rio Grande do Sul, Brasil. Cad Saúde Pública [Internet]. 2003 [acesso 21 jan 2019];19(1):27-34. DOI: 10.1590/S0102-311X2003000100004

15. Brasil. Ministério da Saúde. Política Nacional de Humanização: PNH: Humaniza SUS [Internet] Brasília: Ministério da Saúde; 2015 [acesso 28 fev 2019]. Disponível: https://bit.ly/2WTXvDD

16. Backes DS, Koerich MS, Rodrigues ACRL, Drago LC, Klock P, Erdmann AL. O que os usuários pensam e falam do Sistema Único de Saúde? Uma análise dos significados à luz da carta dos direitos dos usuários. Ciênc Saúde Coletiva [Internet]. 2009 [acesso 2 maio 2019];14(3):903-10. DOI: 10.1590/S1413-81232009000300026

17. Porto $D$, Schierholt SR, Costa AM. Retratos da relação médico-paciente na atenção básica. Rev. bioét. (Impr.) [Internet]. 2012 [acesso 19 ago 2019];20(2):288-99. Disponível: https://bit.ly/3eVs2qL

18. Machado FA, Venturini RR, Manzan ALA, Silva GR. Relação do paciente com o serviço em unidades básicas de saúde sob a óptica dos médicos e dos pacientes. Rev Bras Med Fam Comunidade [Internet]. 2015 [acesso 24 out 2019];10(37):1-11. DOI: 10.5712/rbmfc10(37)773

19. Santiago RF, Mendes ACG, Miranda GMD, Duarte PO, Furtado BMASM, Souza WV. Qualidade do atendimento nas unidades de saúde da família no município de Recife: a percepção dos usuários. Ciênc Saúde Coletiva [Internet]. 2013 [acesso 8 jan 2019];18(1):35-44. DOI: 10.1590/ S1413-81232013000100005

20. Brasil. Ministério da Saúde. Carta dos direitos dos usuários da saúde [Internet]. 2a ed. Brasília: Ministério da Saúde; 2007 [acesso 2 maio 2019]. Disponível: https://bit.ly/30GoVhC

21. Nascimento RCRM, Álvares J, Guerra AA Jr, Gomes IC, Costa EA, Leite SN et al. Disponibilidade de medicamentos essenciais na atenção primária do Sistema Único de Saúde. Rev Saúde Pública [Internet]. 2017 [acesso 3 abr 2019];51(supl 2):10s. DOI: 10.11606/s1518-8787.2017051007062

22. Helfer AP, Camargo AL, Tavares NUL, Kanavos P, Bertoldi AD. Capacidade aquisitiva e disponibilidade de medicamentos para doenças crônicas no setor público. Rev Panam Salud Pública [Internet]. 2012 [acesso 3 abr 2019];31(3):225-32. Disponível: https://bit.ly/2P699Y1

23. Tesser CD, Poli Neto P, Campos GWS. Acolhimento e (des)medicalização social: um desafio para as equipes de saúde da família. Ciênc Saúde Coletiva [Internet]. 2010 [acesso $11 \mathrm{fev}$ 2019];15(supl 3):3615-24. DOI: 10.1590/S1413-81232010000900036

\title{
Participação dos autores
}

Ana Felisa Hurtado Guerrero idealizou o projeto, participou da revisão crítica do artigo e orientou a pesquisa. Ana Possidonio Alves, Gilmara Lima Libório e Joziane Vieira de Freitas idealizaram o projeto e coletaram e organizaram os dados. José Camilo Hurtado Guerrero analisou os dados, interpretou os resultados e colaborou na revisão crítica do texto. Todos os autores redigiram o artigo.

\section{Correspondência}

José Camilo Hurtado Guerrero - Universidade Federal do Oeste do Pará. Instituto de Biodiversidade e Floresta. Rua Vera Paz, s/n, Salé CEP 68040-470. Santarém/PA, Brasil.

\author{
Ana Felisa Hurtado Guerrero - Doutora - guerrerocol1@hotmail.com \\ (iD) 0000-0002-2816-6235 \\ Ana Possidonio Alves - Graduada -ana.coari2016@gmail.com \\ (iD) $0000-0002-6183-8611$ \\ Gilmara Lima Libório - Graduada - gilmaralimaliborio7475@gmail.com \\ (i) $0000-0002-1776-2883$ \\ Joziane Vieira de Freitas - Graduada - joseanevieiradefreitas@gmail.com \\ (iD) 0000-0003-0600-251X \\ José Camilo Hurtado Guerrero - Doutor - jhocamhur@gmail.com \\ (D) $0000-0001-9062-1860$
}

